\title{
Le canal de Suez : un chantier dans le désert
}

The Suez canal: A building site in the desert

\section{Lionel Dufaux}

\section{(2) OpenEdition \\ 1 Journals}

\section{Édition électronique}

URL : http://journals.openedition.org/artefact/3564

DOI : 10.4000/artefact.3564

ISSN : 2606-9245

Éditeur :

Association Artefact. Techniques histoire et sciences humaines, Presses universitaires du Midi

\section{Édition imprimée}

Date de publication : 15 mars 2019

Pagination : 295-304

ISBN : 978-2-8107-0623-5

ISSN : 2273-0753

\section{Référence électronique}

Lionel Dufaux, "Le canal de Suez : un chantier dans le désert », Artefact [En ligne], 9 | 2018, mis en ligne le 04 mars 2020, consulté le 28 novembre 2020. URL : http://journals.openedition.org/artefact/ 3564 ; DOI : https://doi.org/10.4000/artefact.3564

\section{(c) (i) (9)}

Artefact, Techniques, histoire et sciences humaines est mise à disposition selon les termes de la Licence Creative Commons Attribution - Pas d'Utilisation Commerciale - Pas de Modification 4.0 International. 


\section{Le canal de Suez : un chantier dans le désert}

\section{Lionel Dufaux}

\section{Résumé}

Le percement du canal maritime de Suez, entre 1859 et 1869, a été l'un des plus importants chantiers de travaux publics du xix siècle. S'inscrivant dans un temps long, lointain successeur du canal des pharaons, le canal de Suez représente un tour de force technique et une vraie rupture : confronté à d'importantes contraintes géographiques, climatiques et logistiques, le chantier connaît une profonde réorganisation à partir de 1863, visant à lui donner une structuration rigoureuse, faisant largement appel à la mécanisation et constituant un véritable saut dans la modernité grâce à l'emploi de machines innovantes.

\section{Mots-clés}

chantier, construction, travaux publics, voie navigable

99 Lionel Dufaux, « Le canal de Suez : un chantier dans le désert », Artefact, 9, 2018, p. 295-304. 


\section{The Suez canal: A building site in the desert}

\section{Abstract}

The drilling of the Suez maritime canal, between 1859 and 1870, was one of the most important civil engineering construction site of the $19^{\text {th }}$ century. Stakeholder of a long time history, distant successor of pharaohs' canal, the Suez canal is a technical tour de force and a true rupture: confronted with important geographical, logistical and climatic constraints, the construction site has been deeply reorganized from 1863, with a rigorous structuring, important mechanization and the use of innovative machines.

\section{Keywords}

civil engineering, construction site, waterway 


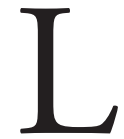

'Exposition universelle de 1867, à Paris, a été l'occasion pour la Compagnie universelle du canal de Suez de proposer une luxueuse mise en scène de l'avancée de l'un des plus importants chantiers de génie civil du XIX ${ }^{e}$ siècle ${ }^{1}$. Derrière la façade égyptisante du pavillon de Suez, les visiteurs pouvaient admirer un diorama représentant le paysage de l'isthme, ainsi que des collections de spécimens géologiques, de la faune et de la flore. L'un des clous de cette présentation était une impressionnante série de plans en relief et de modèles illustrant les machines utilisées sur le chantier ${ }^{2}$. Visant tout autant à démontrer le bon déroulement des travaux et à rassurer les actionnaires tout en soulignant la modernisation de l'Égypte ${ }^{3}$, l'exposition de la Compagnie rappelle le caractère hors normes du chantier. Celui-ci se déroule sur dix années, entre 1859 et 1869 , bien que la formalisation du projet ait été initiée bien plus tôt, et que le canal ait fait l'objet de nombreuses campagnes de travaux après son inauguration, le 20 novembre 1869. Long d'environ $164 \mathrm{~km}$, le canal maritime de Suez devait relier la Méditerranée à la mer Rouge en traversant une région désertique, ponctuée de plusieurs seuils et de lacs d'eau salée.

La construction du canal de Suez s'inscrit à la convergence de l'industrie, de la finance et de la diplomatie. Elle rappelle l'importance de cette entreprise qui constitue, sinon une rupture, du moins un jalon fondamental dans l'évolution des techniques mises en œuvre dans les travaux publics, qu'il s'agisse de la mécanisation ou de l'organisation du travail.

\section{Aux origines du canal de Suez}

Le premier coup de pioche du chantier est donné le 25 avril 1859, sur le site où sera implanté Port-Saïd, qui marque l'entrée du canal côté Méditerranée ${ }^{4}$. Mais l'histoire d'une voie navigable dans l'isthme de Suez est déjà longue de près de deux millénaires : au XIX ${ }^{\mathrm{e}}$ siècle avant notre ère, le

\footnotetext{
1. Nathalie Montel, Le Chantier du canal de Suez, Paris, Presses de l'école nationale des ponts et chaussées, 1998.

2. Catalogue général de l'Exposition Universelle de 1867 à Paris. Deuxième partie (groupes VI à X), Paris, E. Dentu, 1867, p. 289-290.

3. Christiane Demeulenaere-Douyère, "L'Égypte, la modernité et les expositions universelles ", Bulletin de la Sabix. Société des amis de la Bibliothèque et de l'Histoire de l'École polytechnique, 2014, vol. 54, p. 37-41.

4. Ferdinand De Lesseps, Percement de listhme de Suez. Exposé et documents officiels, Paris, Plon, 1855 ; id., Lettres, journal et documents pour servir à l'histoire du canal de Suez, Paris, Plon, 1875.
} 
pharaon Sésostris III aurait en effet ordonné le percement d'un canal. Sans pouvoir en confirmer l'existence, l'archéologie a révélé la présence d'une voie navigable entre le Nil et la mer Rouge, attestée à l'époque de Darius Ir au VI ${ }^{e}$ siècle av. J.-C. Ce " canal des pharaons" semble avoir été désaffecté au VIII ${ }^{e}$ siècle de notre ère. Sans vouloir le considérer comme étant à l'origine du canal de 1869, il inscrit néanmoins la traversée de l'isthme de Suez dans un temps nettement plus long 5 .

Le contrôle de la route commerciale entre l'Europe et les Indes est le principal enjeu d'un canal maritime entre la Méditerranée et la mer Rouge. Évitant le long détour par le cap de Bonne-Espérance, le canal devait permettre aux Français de contrecarrer la domination britannique sur le commerce maritime. L'expédition conduite en Égypte en 1798 sous la direction du général Bonaparte a été l'occasion de décrire cette région mais également d'effectuer une première étude technique menée par JacquesMarie Le Père, directeur des Ponts et Chaussées ${ }^{6}$. Une trentaine d'années plus tard, au début des années 1830, plusieurs savants et ingénieurs, inspirés par le saint-simonisme, ont vu dans le projet d'un canal entre la Méditerranée et la mer Rouge un moyen d'améliorer la fluidité du commerce mondial mais également de rendre le monde "voyageable et habitable comme l'Europe ", d'aménager de valoriser un territoire jusque-là laissé de côté. Plusieurs projets s'opposent : certains visent un tracé au plus court, d'autres veulent intégrer Alexandrie ou Le Caire ${ }^{7}$. Les études de nivellement permettent de constater la très faible différence de niveau entre la Méditerranée et la mer Rouge, autorisant le percement d'un canal sans écluses ${ }^{8}$.

Fin connaisseur du milieu politique égyptien, diplomate longtemps en poste à Alexandrie, Ferdinand de Lesseps entretient des liens de proximité

5. Christiane Ziegler, "Le canal des pharaons de l'Antiquité au Moyen Âge : aux origines du canal de Suez ? ", in Claude Mollard et Gilles Gauthier (dir.), L'épopée du canal de Suez, Paris, Gallimard, 2018, p. 20-25.

6. Jacques Marie Le Père, Mémoire sur la communication de la mer des Indes à la Méditerranée par la mer Rouge et l'isthme de Soueys, Paris, Imprimerie royale, 1815.

7. Alexis Barrault et Émile Barrault, «Le canal de Suez et la question du tracé, les divers projets en présence avec une carte ", Revue des deux mondes, 1856, t. I, p. 70-102 ; Paulin Talabot, "Le Canal des deux mers d'Alexandrie à Suez ", Revue des deux mondes, 1855, t. x, p. 480-536.

8. Philippe RÉGNIER, «Après Bonaparte, mais avant Lesseps : les projets de Prosper Enfantin et des saint-simoniens ", in Claude Mollard et Gilles Gauthier (dir.), L'épopée du canal de Suez, Paris, Gallimard, 2018, p. 32-37. 
avec Mohamed Saïd Pacha, fils du vice-roi Méhémet Ali qui accède au pouvoir en $1854^{9}$. S'appuyant sur les conclusions des ingénieurs, qui valident le principe d'un canal direct sans écluses, Lesseps mène une active campagne diplomatique pour défendre le projet d'une compagnie pour creuser le canal face à l'opposition du Premier Ministre britannique, Lord Palmerston. Lesseps réunit une commission internationale, gage de neutralité, qui confirme les choix techniques, malgré l'hostilité des Britanniques et du Sultan ottoman, dont vice-roi d'Égypte est un vassal. La Compagnie universelle du canal maritime de Suez est constituée le 15 décembre 1858, avec deux sièges sociaux à Paris et Alexandrie; le capital de 200 millions de francs est divisé en actions de 500 francs, dont le versement se fera en sept fois, de 1858 à 1866 . Vingt et un mille souscripteurs répondent à cette immense levée de capitaux : $56 \%$ des actions sont acquises par des petits épargnants français, $25 \%$ sont détenues par le souverain égyptien. Un contrat est conclu avec l'entrepreneur de travaux publics Alphonse Hardon sous le régime de la "régie intéressée ", prévoyant le versement d'une partie des recettes générées par l'exploitation du canal ${ }^{10}$. Le pari d'une voie d'eau en plein désert parcourue exclusivement par des navires motorisés est lancé, et les travaux peuvent commencer.

\section{Un chantier dans le désert}

Le projet du canal de Suez prévoyait une voie de 80 à 100 mètres de large à la ligne d'eau, pour 8 mètres de profondeur. Le volume des terrassements à réaliser est évalué à 75 millions de mètres cubes. Il s'agit alors du premier chantier d'une telle ampleur, un véritable chantier d'exception. La conduite des travaux en plein désert impose d'organiser l'approvisionnement en eau douce, non seulement pour les ouvriers mais également pour les machines : les puits disponibles dans le désert sont insuffisants, et l'on utilise des appareils de dessalinisation qui s'avèrent rapidement peu efficaces. L'eau est alors importée par barques, ce qui impose de longues et pénibles opérations d'acheminement.

À son ouverture, le chantier repose malgré tout, pour l'essentiel, sur des moyens qui peuvent apparaître comme sommaires, voire archaïques.

9. Ghislain de Diesbach, Ferdinand de Lesseps, Paris, Perrin, 2009.

10. N. Montel, Le Chantier du canal de Suez, op. cit. 
Comme l'a souligné Nathalie Montel, la mécanisation des chantiers de travaux publics est restée assez limitée dans la première moitié du XIX ${ }^{e}$ siècle $^{11}$. La mise à disposition de la Compagnie d'une abondante main-d'œuvre par Mohamed Saïd Pacha, prévue dans le contrat, devait pallier cette absence de machines. Embauchée sous le régime de la corvée, qui prévoyait que les fellahs devaient plusieurs journées de travail au vice-roi d'Égypte pour des travaux d'entretien des canaux d'irrigation, cette armée de 25000 ouvriers égyptiens était complétée par de la main-d'œuvre italienne, grecque ou encore allemande. Une vraie tour de Babel encadrée par des contremaitres français, maniant un outillage rudimentaire constitué de pelles, de pioches, de jougs et de couffins. C'est à la force des bras que l'on remplit des couffes de paille tressée et que l'on déverse ensuite pour former des talus. Peu qualifiée, souvent versatile, cette main-d'œuvre reste peu de temps sur le chantier, contribuant à sa désorganisation. Les conditions de travail y sont en effet effroyables, et valent à la ville nouvelle de Port Saïd le surnom de "Cayenne du désert $»^{12}$.

La Compagnie du canal de Suez est rapidement confrontée à d'importances difficultés, relayées avec complaisance par les Britanniques. La répartition des rôles entre la Compagnie et l'entreprise d'Hardon, les recrutements de main-d'œuvre insuffisants ou encore certains choix comme celui de privilégier le percement de la rigole préfigurant le futur canal plutôt que de concentrer les premiers efforts sur le canal d'eau douce pèsent en effet sur la progression du chantier. En 1863, le nouveau vice-roi Ismail Pacha abolit l'esclavage et supprime la corvée, mettant un coup d'arrêt aux travaux. Mais un processus de réorganisation du chantier, opéré depuis quelques mois, allait néanmoins sauver l'entreprise.

\section{La modernisation du chantier}

En avril 1862, le polytechnicien François Philippe Voisin est nommé directeur des travaux. Cet ingénieur des Ponts et Chaussées, présent sur le chantier depuis deux années, va profondément changer l'organisation des travaux. Ses premières mesures concernent l'adoption de méthodes

11. E. Mougel et A. Mouchelet, Mécanique des travaux publics, ou Application de la vapeur des machines les plus modernes à la construction des édifices, chemins de fer, ports, canaux, etc., Paris, Rousseau, 1841.

12. N. Montel, Le Chantier du canal de Suez, op. cit. 
rigoureuses éprouvées sur le terrain des travaux de génie civil en France : Voisin impose la tenue de livres de comptes et de métré, il calcule le rendement des hommes et des machines, hiérarchise et précise les tâches de tous, du simple expéditionnaire jusqu'aux ingénieurs, revoit l'implantation et l'approvisionnement des magasins de matières premières, de matériel et de vivres. Le chantier est découpé géographiquement en quatre divisions, elles-mêmes composées de plusieurs sections, et l'organigramme est adapté aux besoins du chantier. Enfin, le gabarit du canal à la ligne d'eau est réduit, et passe de 100 à 56 mètres ${ }^{13}$ (Fig. 1).



\section{Fig. 1. - A. Broise et Thieffry, « Dragages ", Mémoires et compte rendu de la Société des ingénieurs civils, vol. 17, 1864, pl. 42}

\section{Bibliothèque du Cnam. Cnum - Conservatoire numérique des Arts et Métiers, http://cnum.cnam.fr/}

Le contrat avec Hardon est dénoncé le $1^{\text {er }}$ janvier 1863 : la Compagnie réalise les travaux en régie directe avant de les diviser en lots, confiés à des entrepreneurs français et britanniques. $75 \mathrm{~km}$ ont été creusés, mais il en reste encore 85. Les terrassements du seuil rocheux d'El-Guisr sont pris en charge par Alphonse Couvreux ; la société Dussaud et Frères construira les jetées de Port-Saïd en ciment artificiel ; Lafarge sera le fournisseur de chaux ; l'Écossais Aïton se voit confier les dragages de Port-Saïd au lac Ballah, tandis que Borel, Lavalley et Compagnie assurera ceux entre El-Guisr et la mer Rouge. Tous ces entrepreneurs importent sur le chantier de nombreuses machines mises en mouvement par la force de la vapeur.

13. François Philippe VoIsın, Le Canal de Suez, Paris, Dunod, 1902. 
Cette dernière s'impose pour l'ensemble des travaux : d'abord, avec l'installation de machines à vapeur fixes et de locomobiles qui fournissent la force nécessaire au fonctionnement de certains ateliers ou de machines, animées par l'intermédiaire de courroies ; ensuite, avec l'emploi d'équipements disposant de leur propre moteur à vapeur ; enfin, avec la construction de centaines de kilomètres de voies ferrées légères et démontables, parcourues par des trains à vapeur pour acheminer des matériaux et évacuer les déblais. Ces machines doivent être suffisamment robustes et régulièrement entretenues, graissées et révisées pour fonctionner de manière optimale dans le désert, avec un environnement marqué par la poussière de sable et de forts écarts de températures. Voisin est également attentif à ce que l'alimentation en eau douce et l'approvisionnement de combustible soient continus (Fig. 2).

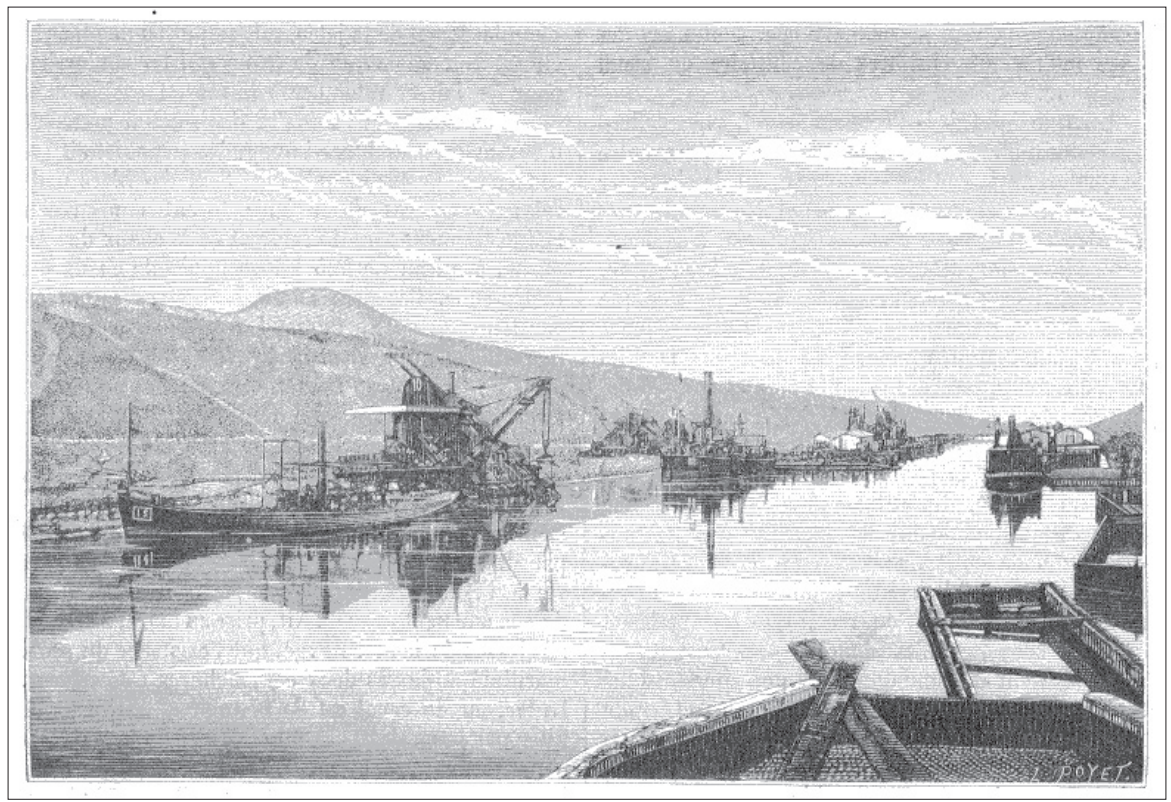

Fig. 2. - Louis Poyet, « Creusement du canal maritime de Suez. L'excavateur Couvreux dans la grande tranchée d'El-Guisr »

Gravure publiée dans La Nature, $10^{\mathrm{e}}$ année, $1^{\mathrm{er}}$ semestre, 1882, p. 344.

Bibliothèque du Cnam. Cnum - Conservatoire numérique des Arts et Métiers, http://cnum.cnam.fr 
Le chantier de Suez est l'occasion d'utiliser des machines jusqu'alors peu employées voire inédites. Pour les déblais d'El-Guisr, Alphonse Couvreux utilise son excavateur à godets, breveté en 1860. Cette machine comporte une chaîne animant des godets métalliques qui arrachent les morceaux de roche et les déposent dans des wagons en vue de leur évacuation. La machine, pourvue de sa propre chaudière, se substitue aux terrassiers et à leurs brouettes (Fig. XVII, cahier couleur). En trois ans, Couvreux arrache ainsi près de 9 millions de mètres cubes de roche dure sur 15 kilomètres. Pour les travaux de dragage, c'est-à-dire de terrassements sous l'eau, la société Borel, Lavalley et Compagnie utilise des dragues et des élévateurs de dimensions jusque-là inconnues, construits notamment Ernest Gouin et les Forges et Chantiers de la Méditerranée. Installées sur les rives ou sur des barges, ces machines creusent le fond de la rigole et évacuent les déblais en formant des talus (Fig. XVIII, cahier couleur). À cela s'ajoutent de multiples grues, des broyeurs, des plans inclinés, fonctionnant avec des machines à vapeur.

En 1867, on compte soixante-huit dragues à vapeur sur le chantier. À la fin de l'année, Léon Monteil, ingénieur en charge du matériel, évalue la puissance installée à près de 18000 chevaux pour une consommation mensuelle de charbon dépassant les $12000 \mathrm{t}^{14}$. L'accélération des travaux entraîne une forte augmentation des coûts, et l'intervention de Napoléon III s'avère nécessaire pour que Lesseps puisse maintenir la compagnie à flot avec un emprunt de 100 millions de francs.

Le 18 mars 1869, les eaux de la Méditerranée rejoignent les lacs Amers, suivies, le 16 août, des eaux de la mer Rouge. L'inauguration du canal donne lieu à plusieurs jours de festivités, entre le 17 et le 20 novembre 1869, présidées par l'impératrice Eugénie. Mais il faut attendre le 15 avril 1871 pour voir l'achèvement des terrassements.

Par son ampleur et l'importance de la mécanisation, le chantier du canal de Suez marque une étape majeure dans l'histoire des techniques et des travaux publics. Si l'emploi de la vapeur et de nombreuses machines est tout à fait significatif, il est ici corrélé à l'organisation même du chantier et sa très forte structuration, en particulier après 1863. L'histoire des

14. Léon Monteil, Percement de l'isthme de Suez : description des travaux et ouvrages d'art définitifs, des machines et des appareils mis en cuvre sur les chantiers, des procédés et du matériel employés pour l'exploitation du canal maritime, Paris, Bureaux des annales industrielles, 1875. 
techniques est ici profondément liée, comme c'est souvent le cas, aux questions économiques et financières, mais également à des problématiques géographiques, politiques et diplomatiques.

Le canal de Suez est, à cet égard, particulièrement éclairant. Il s'agit d'un chantier quasi perpétuel, qui a nécessité la mise au point de machines toujours plus performantes pour maintenir l'infrastructure en état de fonctionnement, mais aussi pour l'adapter à l'évolution du trafic maritime. Le canal a ainsi fait l'objet de plusieurs campagnes de travaux qui lui ont permis d'atteindre une largeur moyenne d'environ $345 \mathrm{~m}$ pour une profondeur de $24 \mathrm{~m}$. La nationalisation par Gamal Abdel Nasser en 1956, et sa fermeture en 1967 après la guerre des Six Jours rappellent la dimension géopolitique du canal de Suez.

\section{L'auteur}

Lionel Dufaux est ingénieur d'études, responsable de collections au musée des Arts et Métiers (Conservatoire national des arts et métiers). Docteur en histoire de l'EHESS, il s'intéresse notamment à l'histoire des transports, des musées et des collections techniques. Il a récemment publié L’Amphithéâtre, la galerie et le rail. Le Conservatoire des arts et métiers et ses collections au xix siècle (Presses universitaires de Rennes, 2017) et "L'objet vélo, de la draisienne au Vélib' », À bicyclette ! (Silvana Editoriale/Historial de la Vendée, 2018).Contact : lionel.dufaux@ lecnam.net 